\title{
INVESTIGACIONES
}

\section{La alfabetización tecnológica: de la informática al desarrollo de competencias tecnológicas*}

\author{
Technological literacy: from computing to the \\ development of technological competences
}

\author{
Diego González Campos, ${ }^{a}$ Fredy Olarte Dussán, ${ }^{b}$ Javier Corredor Aristizabal ${ }^{a c}$ \\ aDepartamento de Psicología, Universidad Nacional de Colombia \\ Telf.: (57) 3057081083. Correo electrónico: diagonzalezca@ gmail.com \\ ${ }^{\text {b}}$ Departamento de Ingeniería Eléctrica y Electrónica, Universidad Nacional de Colombia \\ Correo electrónico: faolarted@unal.edu.co \\ 'Telf.: (57) 3165000. Correo electrónico: jacorredora@unal.edu.co
}

\begin{abstract}
RESUMEN
La alfabetización tecnológica es necesaria para aumentar la competitividad y productividad de la población, pero la organización actual del sistema educativo escolar no facilita el desarrollo de tales habilidades y competencias. Este estudio indaga los efectos que tiene el uso de una metodología de aprendizaje basada en proyectos con uso de Tecnologías de la Información y la Comunicación TIC en la alfabetización tecnológica de los estudiantes de diversos colegios. Se recopila información de 6 profesores y 95 alumnos de cinco municipios de Colombia. La información es analizada de manera cualitativa por medio de un proceso de triangulación que contempla categorías preestablecidas y emergentes. Los resultados indican que la estrategia tiene efectos sobre diversos aspectos del ambiente de aprendizaje: rol docente, interacción entre estudiantes, clima de relaciones, significatividad y competencias tecnológicas. Se concluye que la estrategia de aprendizaje implementada es efectiva para lograr la alfabetización tecnológica.
\end{abstract}

Palabras clave: educación en tecnología, aprendizaje basado en proyectos, aprendizaje de diseño, ambientes de aprendizaje, aprovechamiento escolar.

\begin{abstract}
Technological literacy is a need for greater competitiveness and productivity in the population. But the current organization of school educational system does not facilitate the development of such skills and competences. The purpose of this research is to identify the effects stemmed from the implementation of a project-based learning program with use of informatics and communication technologies in the technological literacy of students from different public schools. It gathers information of 6 teachers and 95 students of five municipalities in Colombia. The Information is analyzed trough qualitative methods by a triangulation process which provides pre and emerging categories. Results suggest that the program has effects on different aspects of the learning environment: teacher role, student's interaction, relations environment, meaningfulness and technological competences. It concludes that the training program that was implemented is effective to achieve technological literacy.
\end{abstract}

Key words: technology education, project based-learning, design learning, learning environments, learning achievement.

Esta investigación es parte de la agenda del Centro de Innovación Educativa Regional (CIER-centro) y fue financiada con recursos del Programa Colciencias n 38453 de Ciencia, Tecnología e Innovación (CTEI) en innovación educativa con uso de las tecnologías de la información y la comunicación TIC. 


\section{INTRODUCCIÓN}

En la actualidad es necesario lograr que los estudiantes sean capaces de usar, manejar, evaluar y entender la tecnología (ITEA/ITEEA, 2000, 2002, 2007). No obstante, el aprendizaje conseguido en esta materia ha sido limitado, debido a que la visión que se tiene sobre la naturaleza de la tecnología ha estado ligada principalmente a la ofimática (Jones, Buntting \& de Vries, 2013). De la misma manera, los efectos del cambio pedagógico derivado de la tecnología se han condicionado, en la mayor parte de los casos, a ganancias en la transmisión de contenidos, pero no a otros elementos importantes como la significatividad, el rol docente, la interacción entre estudiantes, el clima de relaciones y las competencias tecnológicas. Los retos de la alfabetización tecnológica implican cambios en las concepciones de los estudiantes y profesores sobre la naturaleza y los efectos de la tecnología. Es por ello necesario facilitar nuevos espacios de aprendizaje que permitan al estudiante desarrollar las habilidades necesarias para la resolución de problemas a través del uso de la misma.

La introducción de metodologías de Aprendizaje Basadas en Proyectos (ABP) en la enseñanza de la tecnología permite que los estudiantes aprendan en la práctica cotidiana, facilitando que estos lleven a cabo acciones de diseño, exploración, identificación de problemas, construcción, modelamiento, reparación y evaluación en contextos de aprendizaje significativos (Ministerio de Educación Nacional [MEN], 2008). Además, este tipo de metodologías ha demostrado tener efectos positivos sobre el desempeño de los estudiantes en la evaluación de contenidos de conocimiento (Panasan \& Nuangchalerm, 2010), la responsabilidad, la auto-dirección, la comunicación, la creatividad (Wurdinger \& Qureshi, 2015), la motivación (Acar, 2013) y el trabajo colaborativo (Lee, Huh \& Reigeluth, 2015).

Sin embargo, el éxito de esta metodología depende de que los estudiantes tomen responsabilidad de su proceso de aprendizaje, se definan objetivos claros, realicen procesos de monitoreo, reflexión y sostengan la motivación (English \& Kitsantas, 2013). Existe evidencia que demuestra que lo anterior no siempre sucede, porque adaptarse a la estructura del ABP implica un reto tanto para estudiantes como para profesores (Krajcik, et al., 1994; Krajcik et al., 1998).

Con el fin de lograr que los alumnos se desenvuelvan exitosamente en el ABP, es necesario contar con ambientes de aprendizaje y prácticas de enseñanza que soporten el aprendizaje auto-regulado de los estudiantes (English \& Kitsantas, 2013). En efecto, se ha demostrado que los ambientes de aprendizaje centrados en el estudiante generan un mayor compromiso emocional en los alumnos y una menor dependencia hacia el docente (Wu \& Huang, 2007). Además, dichos ambientes facilitan el desarrollo de procesos en los estudiantes tales como la resolución de problemas, el razonamiento divergente y el pensamiento crítico (Hannafin \& Land, 1997).

Este estudio pretende mostrar, a partir de datos cualitativos, que el uso de una metodología de ABP en la enseñanza de la tecnología modifica los ambientes de aprendizaje en varios sentidos: trasladando la práctica pedagógica de un énfasis en el docente a un énfasis en el estudiante, haciendo que los docentes asuman un rol mediador y facilitador, logrando promover interacciones entre estudiantes de tipo colaborativo, instalando un clima de relaciones horizontal entre profesores y alumnos, promoviendo en los estudiantes aprendizajes significativos para la solución de problemas de un contexto real y desarrollando competencias tecnológicas en el uso y apropiación de la tecnología y la solución de problemas. 


\section{LA EDUCACIÓN EN TECNOLOGÍA Y LA ALFABETIZACIÓN TECNOLÓGICA}

Los avances en ciencia y tecnología y sus impactos sobre el funcionamiento de la sociedad han puesto de manifiesto la necesidad de replantear el sistema educativo y los objetivos del mismo, para hacerlos acordes a las realidades cotidianas de los estudiantes. Debido a ello, se han venido desarrollando diferentes directrices sobre lo que debe ser la educación escolar, dando paso a un nuevo sistema de competencias y currículo escolar que promueve el uso y apropiación de la tecnología como una herramienta fundamental en la sociedad actual (Ver Australian Education Council, 1994; de Vries, 1999; Qualifications and Curriculum Authority, 2000). Tales lineamientos curriculares han estado orientados a la promoción de la educación en tecnología principalmente en siete sentidos: desarrollo de habilidades, artes industriales y entrenamiento vocacional, tecnología de la información de diseño, tecnología como una ciencia aplicada, tecnología integrada con la ciencia, la ingeniería y las matemáticas, las múltiples tecnologías y la alfabetización tecnológica (Jones, et al., 2013; Rasinen, 2003).

No resulta fácil llegar a un acuerdo sobre lo que significa la alfabetización tecnológica, debido a la multiplicidad de visiones, intereses y expectativas que giran alrededor de la misma (Devlin, Feldhaus \& Bentrem, 2013). Sin embargo, un punto de encuentro entre las diferentes perspectivas es el desarrollo de habilidades que les permitan a los individuos resolver problemas, proponer soluciones y tomar decisiones a través de su capacidad para comprender, evaluar, usar y transformar objetos, procesos y sistemas tecnológicos (MEN, 2008), lo cual además implica el despliegue de tres dimensiones interdependientes: conocimiento, formas de pensar y capacidad para actuar (ITEA/ITEEA, 2000, 2002, 2007).

Diferentes países han asumido el reto de la alfabetización tecnológica, generando así planes curriculares que establecen competencias y desempeños específicos sobre lo que se espera aprenda el estudiante en materia de tecnología. Entre dichos objetivos se encuentran lograr que el estudiante comprenda la historia de la tecnología, conozca los efectos de la tecnología sobre la naturaleza y sea capaz de comprender, manejar y alterar los elementos tecnológicos de su entorno para dar solución a distintas problemáticas (Rasinen, 2003).

En Colombia, de acuerdo con los lineamientos del Ministerio de Educación Nacional (MEN) son cuatro las competencias que definen la alfabetización de los estudiantes en el área de tecnología. La primera es naturaleza y evolución de la tecnología y hace referencia al conocimiento de los conceptos, características y objetivos fundamentales de la disciplina, así como su historia y evolución. La segunda competencia es apropiación y uso de la tecnología, y busca que los estudiantes sean capaces de hacer uso de las herramientas tecnológicas de manera adecuada y crítica, con el objetivo de optimizar y aumentar la productividad en la realización de diferentes tareas. La tercera competencia es solución de problemas con tecnología, y consiste en la capacidad de diseñar estrategias para la identificación, formulación y solución de problemas con tecnología. La última competencia es tecnología y sociedad, la cual aborda temáticas relacionadas con las actitudes de los estudiantes hacia la tecnología, el reconocimiento del potencial de los recursos, la evaluación de los procesos, el análisis de sus impactos, la ética, la responsabilidad social, la comunicación y la interacción social. La suma de todas estas competencias tiene como objetivo la consolidación de un campo interdisciplinario que rompa la brecha entre el conocimiento tecnológico y la vida cotidiana, y permita contribuir a la competitividad y productividad de la población (MEN, 2008). 
Según el MEN (2008), ser competente en el uso y apropiación de la tecnología mejora la competitividad y productividad de la población, ya que se desarrollan habilidades que permiten la solución de problemas a través del uso de la misma. No obstante, el desarrollo de dichas habilidades requiere un cambio en los ambientes de aprendizaje tradicionales. Es necesario contar entonces con una metodología de enseñanza que permita desarrollar efectivamente dichas competencias, más allá de cambios secundarios en el discurso y las actividades de clase.

\section{AMBIENTES DE APRENDIZAJE}

Dos vertientes teóricas que han profundizado en el aprendizaje han sido el enfoque conductista y el enfoque constructivista. El primero establece que los conocimientos pueden ser transferidos por los profesores o transmitidos a través de la tecnología y adquiridos por los alumnos. El segundo parte de una visión en la cual el conocimiento es elaborado individual y socialmente por los estudiantes, fundado en experiencias propias y representaciones del mundo y sobre las bases de los conocimientos declarativos ya conocidos (Jonassen, 2000). Del enfoque conductista se derivan los ambientes de aprendizaje centrados en el profesor, los cuales se fundamentan sobre las bases de la disciplina y el control, y en donde la conformidad y el aprendizaje pasivo son privilegiados por encima de la iniciativa y el aprendizaje activo (Freiberg, 1999). Por ello, las tareas o actividades empleadas en este modelo se enfocan en mantener el control sobre los estudiantes y son ejercicios principalmente teóricos. Por ejemplo, las lecturas, las discusiones guiadas y los libros guías son formas de instrucción que por sí mismas ponen al profesor al frente de la clase mientras que todos los estudiantes trabajan en la misma tarea. Se sabe por demás que esta estructura de trabajo limita la participación de los estudiantes y enfoca la clase en los contenidos y no sobre el proceso mismo de aprender (Edwards, 2004). Además, este tipo de actividades se fundamentan en la motivación extrínseca, pues los estudiantes mantienen un buen comportamiento solo a partir de incentivos externos como por ejemplo una buena calificación (Smith, Brabander \& Martens, 2014).

Por otra parte, del enfoque constructivista se derivan los ambientes de aprendizaje centrados en el estudiante. En este los estudiantes tienen un rol activo y enfrentan tareas que se encuentran en un contexto rico y autentico a través de un aprendizaje auto-regulado, son ellos mismos quienes establecen sus objetivos y toman responsabilidad de su proceso de aprendizaje (Smith et al., 2014). Los ambientes de aprendizaje centrados en el estudiante incluyen múltiples fuentes de información, promueven la interacción entre alumnos y modifican la relación alumno-profesor. Todo ello permite más interacciones que van en pro de la creación de significado, el pensamiento reflexivo, la investigación, la discusión exploratoria y la creación de comunidades de aprendizaje (Garret, 2008). Además, el conocimiento en estos ambientes es construido por profesores y alumnos, las fuentes de motivación son intrínsecas y se basan en el diseño de actividades y metodologías que por sí mismas le puedan interesar al estudiante (Ames, 1992).

\section{APRENDIZAJE BASADO EN PROYECTOS}

El aprendizaje basado en proyectos es un modelo instruccional centrado en el estudiante que forma parte de los entornos de aprendizaje constructivistas (Esteban, 2002). Esta me- 
todología representa una forma de aprendizaje situado que permite al estudiante ejercer un rol activo en la adquisición de los conceptos de una asignatura por medio del trabajo en proyectos. En estos proyectos los estudiantes se ven inmersos en problemas significativos y reales que son importantes para ellos y que implican el desarrollo de actividades de diseño e investigación, resolución de problemas y toma de decisiones (Reverte et al., 2007).

En la secuencia didáctica del ABP existen espacios en los cuales se permite a los estudiantes investigar nuevas preguntas, proponer hipótesis y explicaciones, discutir opiniones, enfrentar las propuestas de otros y probar nuevas ideas (Krajcik \& Blumenfeld, 2006). Estos espacios se caracterizan por presentar tareas abiertas, a partir de las cuales se permite la exploración de múltiples caminos, propiciando así la innovación y la invención, por lo cual el énfasis del docente en la planeación y desarrollo de las clases debe pasar de un enfoque en los contenidos y metas a uno centrado en los estudiantes y sus procesos de adquisición y de construcción de conocimiento (Esteban, 2002).

$\mathrm{El} \mathrm{ABP}$ promueve espacios que generan una motivación intrínseca en los estudiantes, estimulan el trabajo cooperativo y colaborativo y permiten la inclusión de elementos que hacen parte de la vida cotidiana de los alumnos (Heydrich, Rojas \& Hernández, 2012). Según Ames (1992) esta estructura de clase crea objetivos enfocados en el dominio real y autónomo de los contenidos, los cuales respaldan el desarrollo de nuevas habilidades, mejoran los niveles de competencia y promueven un esfuerzo por la comprensión. En esa línea, Neo \& Neo (2009) muestran que aquellos estudiantes que trabajan bajo una metodología de ABP mejoran sus habilidades de trabajo en equipo, comunicación y presentación, y además aumentan su interés por las clases y desarrollan su pensamiento crítico.

Según English \& Kitsantas (2013) el desarrollo de tales habilidades depende de que los estudiantes cuenten con la capacidad de auto-regular y auto-dirigir su aprendizaje, para lo cual se debe contar con actividades que permitan un aprendizaje emocional y afectivo, vinculado al contexto y experiencias previas de los estudiantes y que pueda ser usado para resolver problemas reales. Además, se debe implementar un proceso de acompañamiento docente que permita conocer el progreso de los estudiantes a través de los diferentes niveles del proyecto (Thomas, 2000).

Esta metodología implica un reto para estudiantes y docentes (Lee et al., 2013). Los estudiantes deben tomar responsabilidad de su aprendizaje y participar en los procesos de construcción de conocimiento y creación de significado (English \& Kitsantas, 2013), siendo necesario modificar las estructuras de clase tradicional que se encuentran interiorizadas en la mentalidad de los estudiantes y que los ubican en una posición pasiva frente al conocimiento (Hung, 2011). Del mismo modo, los profesores deben abrirse a la perspectiva pedagógica centrada en el estudiante y poseer la motivación y habilidades para poner en uso una nueva estrategia instruccional (Tamim \& Grant, 2013). De esta forma, el ABP requiere que los profesores superen una tendencia extendida a no implementar modelos de enseñanza alternativos (Vratulis et al., 2011), en parte porque muchos no tienen la experticia necesaria para apoyar a los estudiantes en la investigación, para ayudarlos a explorar áreas que no necesariamente son familiares para los docentes (Grant \& Hill, 2006) o para modificar sus concepciones pedagógicas anteriores (Castañeda \& Corredor, 2014).

De la reflexión sobre estas dificultades, experiencias y oportunidades se deriva la estrategia desarrollada. Se plantea así un proceso de acompañamiento continuo, en donde los docentes y estudiantes tienen la oportunidad de reflexionar sobre los diferentes 
fenómenos que acontecen en el aula de clase y aportan información relevante para el reajuste y acomodación del proyecto en el aula.

\section{METODOLOGÍA DE TRABAJO}

En este estudio participaron 6 profesores y 95 estudiantes pertenecientes a cinco instituciones educativas ubicadas en municipios del departamento de Cundinamarca (Soacha, Tabio, Madrid, Villeta) y la ciudad de Bogotá D.C. Estas escuelas participaron en el programa "Diseño de estrategias de enseñanza-aprendizaje basadas en enfoque cognitivo constructivista que integra elementos TIC para el desarrollo de competencias tecnológicas en grados décimo y undécimo", que tenía por objetivo fortalecer las competencias tecnológicas de los estudiantes de grado décimo y undécimo por medio del uso educativo de herramientas TIC. Los profesores participaron en un programa dirigido a implementar la estrategia de enseñanza-aprendizaje basada en proyectos diseñada por el grupo de investigación (Ver Tabla 1). Para cada una de las sesiones los docentes tuvieron un acompañamiento en el proceso de planeación, un acompañamiento en las sesiones de aprendizaje y un encuentro con la comunidad de aprendizaje.

Tabla 1. Fases y etapas de la secuencia didáctica del Aprendizaje Basado en Proyectos

\begin{tabular}{|c|c|c|c|c|c|c|}
\hline Fases & \multicolumn{6}{|c|}{ Etapas } \\
\hline $\begin{array}{c}\text { Fase } 1 \\
\text { Identificación del } \\
\text { problema }\end{array}$ & $\begin{array}{l}\text { Contextualización y } \\
\text { motivación } \\
\text { Presentar: etapas, } \\
\text { plataforma virtual, } \\
\text { estrategia evalu- } \\
\text { ación } \\
\text { Exploración pre- } \\
\text { liminar del proble- } \\
\text { ma con estudiantes }\end{array}$ & \multicolumn{2}{|c|}{$\begin{array}{l}\text { Definición de } \\
\text { fases del proyecto } \\
\text { Definición del } \\
\text { problema } \\
\text { antes) } \\
\text { Asignación } \\
\text { roles }\end{array}$} & \multicolumn{2}{|c|}{$\begin{array}{l}\text { Análisis de alternati- } \\
\text { vas de solución }\end{array}$} & $\begin{array}{l}\text { Evaluación de las } \\
\text { presentaciones }\end{array}$ \\
\hline $\begin{array}{c}\text { Fase } 2 \\
\text { Diseño y } \\
\text { planificación }\end{array}$ & $\begin{array}{l}\text { Presentación selec- } \\
\text { ción de materiales } \\
\text { Definición de mate- } \\
\text { riales }\end{array}$ & $\begin{array}{l}\text { Clasifica } \\
\text { conceptc } \\
\text { cimiento }\end{array}$ & $\begin{array}{l}\text { ación de } \\
\text { os y cono- } \\
\text { s }\end{array}$ & \multicolumn{2}{|c|}{$\begin{array}{l}\text { Presentación diseño } \\
\text { cronograma } \\
\text { Actividad diseño } \\
\text { cronograma } \\
\text { Presentación avance } \\
\text { del proyecto }\end{array}$} & $\begin{array}{l}\text { Presentación herra- } \\
\text { mienta diseño CAD } \\
\text { y simuladores } \\
\text { Diseño de planos, } \\
\text { modelos o dia- } \\
\text { gramas }\end{array}$ \\
\hline $\begin{array}{c}\text { Fase } 3 \\
\text { Ejecución o } \\
\text { Implementación }\end{array}$ & \multicolumn{2}{|c|}{$\begin{array}{l}\text { Preparación y alistamiento de } \\
\text { materiales y algoritmos }\end{array}$} & \multicolumn{2}{|c|}{$\begin{array}{l}\text { Ensamblaje de } \\
\text { piezas u organi- } \\
\text { zación de algoritmos } \\
\text { de programación }\end{array}$} & \multicolumn{2}{|c|}{$\begin{array}{l}\text { Prueba de funcionamiento entre } \\
\text { diversos sistemas }\end{array}$} \\
\hline $\begin{array}{c}\text { Fase } 4 \\
\text { Evaluación y } \\
\text { mejora }\end{array}$ & \multicolumn{2}{|c|}{$\begin{array}{l}\text { Evaluación de funcionamien- } \\
\text { to }\end{array}$} & \multicolumn{2}{|c|}{$\begin{array}{l}\text { Pruebas de funcio- } \\
\text { namiento }\end{array}$} & \multicolumn{2}{|c|}{$\begin{array}{l}\text { Implementación de sugerencias } \\
\text { y mejoras }\end{array}$} \\
\hline
\end{tabular}


El programa implementado tenía como objetivo promover los siguientes rasgos del ambiente de aprendizaje: significatividad afectiva, estructural y pragmática, rol docente mediador-facilitador (Oxfam, 2009), interacción entre estudiantes de tipo colaborativo (Fox-Turnbull, 2012; Muñoz et al., 2014; Rugeles et al., 2014), clima de interacciones integrador-promotor (Madrigal et al., 2011), desarrollo de competencias tecnológicas asociadas al uso y apropiación de la tecnología y la solución de problemas (Bloom, 1956; Marzano, 2001) (Ver Tabla 2):

Tabla 2. Rasgos del ambiente de aprendizaje promovidos por la secuencia didáctica del Aprendizaje Basado en Proyectos

\begin{tabular}{|c|c|c|}
\hline \multicolumn{2}{|c|}{ Características } & Descriptores \\
\hline \multirow{3}{*}{ Significatividad } & Afectiva & $\begin{array}{l}\text { Generar un aprendizaje que se encuentre vinculado emocional } \\
\text { y afectivamente en los estudiantes }\end{array}$ \\
\hline & Estructural & $\begin{array}{l}\text { Promocionar un aprendizaje que se vincule a las experiencias } \\
\text { y conocimientos previos y contextuales de los estudiantes }\end{array}$ \\
\hline & Pragmática & $\begin{array}{l}\text { Integrar el conocimiento en los comportamientos de los } \\
\text { estudiantes, particularmente en aquellos con los que resuelven } \\
\text { situaciones reales }\end{array}$ \\
\hline Rol Docente & $\begin{array}{l}\text { Mediador- } \\
\text { Facilitador }\end{array}$ & $\begin{array}{l}\text { Promover un rol docente de naturaleza interestructurante, } \\
\text { basado en la mediación a través de la pregunta reflexiva y la } \\
\text { orientación participativa de las actividades del ambiente de } \\
\text { aprendizaje }\end{array}$ \\
\hline $\begin{array}{l}\text { Interacción } \\
\text { entre estudiantes }\end{array}$ & Colaborativo & $\begin{array}{l}\text { Crear ambientes de aprendizaje que lleven a los estudiantes a } \\
\text { trabajar en grupo, permitiendo que estos puedan alternar } \\
\text { roles y funciones en torno al logro de objetivos comunes de } \\
\text { aprendizaje }\end{array}$ \\
\hline $\begin{array}{l}\text { Clima de } \\
\text { relaciones }\end{array}$ & $\begin{array}{l}\text { Integrador- } \\
\text { Promotor }\end{array}$ & $\begin{array}{l}\text { Permitir un ambiente en el cual se establezca un clima de } \\
\text { confianza, que invita a expresar opiniones y sentimientos, y } \\
\text { en el que las personas se apoyen mutuamente y se estimulen } \\
\text { o resalten las acciones meritorias }\end{array}$ \\
\hline \multirow{2}{*}{$\begin{array}{l}\text { Competencias } \\
\text { Tecnológicas }\end{array}$} & $\begin{array}{l}\text { Uso y } \\
\text { Apropiación de } \\
\text { la tecnología }\end{array}$ & $\begin{array}{l}\text { Lograr que los estudiantes hagan uso seguro y eficiente de las } \\
\text { herramientas tecnológicas, teniendo en cuenta principios de } \\
\text { funcionamiento y criterios de selección }\end{array}$ \\
\hline & $\begin{array}{l}\text { Solución de } \\
\text { Problemas }\end{array}$ & $\begin{array}{l}\text { Desarrollar habilidades que permitan a los estudiantes } \\
\text { resolver problemas tecnológicos, teniendo conciencia para } \\
\text { elegir la mejor solución }\end{array}$ \\
\hline
\end{tabular}


La información analizada se recolectó a través de 12 grupos focales que fueron llevados a cabo en varios momentos del proyecto. En estos, los docentes exponían sus perspectivas acerca de los elementos del ambiente de aprendizaje generado en el desarrollo de sus clases. Los grupos focales tuvieron una duración en promedio de 25 minutos. Por otra parte, se desarrollaron 16 grupos focales con estudiantes. En dichos grupos se indagaba por el aprendizaje de los estudiantes y las competencias y desempeños tecnológicos que habían desarrollado en la clase de tecnología. La duración de estos grupos focales fue de 15 minutos en promedio. Los datos fueron analizados siguiendo un sistema de codificación estructurado (Namey et al., 2008) y basado en hipótesis (Miles, Huberman \& Saldaña, 2013), los cuales se derivan de las preguntas de los grupos, la literatura previa y las hipótesis que se tenían sobre los efectos del programa. No obstante, el proceso de codificación no se limitó a dichos esquemas, sino que se aplicaron procedimientos de codificación y recodificación ante el surgimiento de nueva información (Saldana, 2009). En el proceso de codificación se hizo una triangulación de fuentes y pares del 60\% de la información (Patton, 2001).

\section{RESULTADOS}

El proceso de codificación y transcripción de los grupos focales de los docentes y estudiantes permitió identificar el efecto del uso de la estrategia de ABP sobre los diferentes rasgos del ambiente de aprendizaje, dicho efecto se organizó en cinco categorías: rol docente, interacción entre estudiantes, clima de relaciones, significatividad y competencias tecnológicas.

\subsection{ROL DOCENTE}

La implementación de la metodología de ABP requiere que los docentes adquieran un rol mediador y facilitador en las actividades de clase, ya que esto permite que los estudiantes participen activamente en la construcción del conocimiento y encuentren un apoyo ante las dificultades. Por ello, es necesario que los profesores abandonen las actividades basadas en la concepción del docente como dueño del conocimiento y asuman una relación más cercana a los estudiantes, la cual se centra en un diálogo para la construcción conjunta del conocimiento. El siguiente ejemplo evidencia la percepción de un docente acerca de su rol dentro del proceso:

"La experiencia del proyecto que es cambiar un poco la película en donde el que proponía era yo, el que llevaba era yo, el que dirigía era yo, etc., y darle la posibilidad a los estudiantes más propositiva, mucho más propositiva, eh eso me ha hecho que en las, en las, en los otros niveles, en los grados décimos, novenos, octavos yo me siente a pensar y a hablar con los estudiantes desde la perspectiva del proyecto. $Y$ me ha resultado mиy constructiva la cosa y muy reveladora porque se sale uno como del esquema un poquitico cuadriculado en donde uno ya tenía el currículo metido aquí en la cabeza y sabía qué hacía con un grado y otro grado”. (docente, grupo focal).

Este fragmento ilustra cómo las características de la metodología de ABP generan una reflexión en los docentes que los lleva a cuestionar y modificar los roles que se dan en su clase, y a su vez, producen un descentramiento docente. Todo ello los lleva a asumir un 
papel de mediación y facilitación del conocimiento que permite a los estudiantes solucionar los problemas a los cuales se enfrentan y desarrollar ciertas habilidades de pensamiento superior. En uno de los foros desarrollados con los docentes, un profesor destacó la importancia de entablar diálogos con los estudiantes que permitan encontrar soluciones a los problemas que estos enfrentan y mantener su motivación en el aula de clase:

"Si, eh no es conocer, no significa que uno sepa el conocimiento que el estudiante está requiriendo, sino que uno sea capaz de conducirlo a que lo tengan porque esa es una de las grandes dificultades. Muchas veces ellos plantean problemas en un universo que no corresponde al universo que uno domina pero en aras a mantener la motivación del estudiante, pues uno se acerca a esa dificultad que el estudiante, o a eso que uno descubre como un problema, y con él, junto con él, trata de darle los elementos para que obtenga lo que requiere para la solución del problema o al menos para aclararlo, caracterizarlo, especificarlo isin que uno necesariamente! sea un experto en eso que él plantea". (docente, grupo focal).

El docente asume así una orientación participativa en las actividades del ambiente de clase, motivando y orientando los estudiantes para que formen comunidades de aprendizaje en las cuales se propicien procesos de investigación y creatividad. De esta manera, se instala en el aula la percepción de los estudiantes como agentes activos en la construcción del conocimiento, posibilitando actividades de aprendizaje definidas por sí mismos y no por el profesor. Uno de los alumnos que hizo parte de los grupos focales, muestra la capacidad de auto-regulación que desarrollan los estudiantes bajo la metodología de ABP:

“...porque es que uno, tiene que, jehh!, el docente solo tiene que, o sea siento que el docente solo tiene que estar como para aclarar si, pero que el conocimiento lo genere uno, yo adquirí un conocimiento, no tengo que depender, si necesito ayuda del docente, ahí sí lo llamo, pero no tengo que estar diciendo que el docente tiene que estar encima mío". (estudiante, grupo focal).

Estos resultados indican que la implementación de la estrategia de ABP permite que se genere un ambiente de clase centrado en los estudiantes, en el cual el docente ocupa una posición secundaria con respecto al conocimiento. No obstante, el rol del docente no desaparece del ambiente de clase, por el contrario, se reclama su presencia a través de un proceso de acompañamiento que asegure la consecución de los objetivos del proyecto.

\subsection{INTERACCIÓN ENTRE ESTUDIANTES}

Es necesario que en un ambiente de aprendizaje centrado en los estudiantes se permita que estos trabajen en equipo y puedan alternar sus roles y funciones en torno al logro de objetivos comunes de aprendizaje. No obstante, tal objetivo enfrenta diversas dificultades. En la información recopilada en los grupos focales de los docentes se evidencia que estos perciben una dificultad inicial en los estudiantes para trabajar de manera colaborativa, la cual podría estar mediada por la existencia de modelos de trabajo individualistas que persisten en la cultura. Ello exige al docente la elaboración de diferentes actividades que permitan a los estudiantes comprender el concepto de trabajo colaborativo y los motiven a sumergirse en él: 
“...la cuestión es enfocarlos para que ellos realmente trabajen de manera colaborativa, porque la cultura está en que ellos, siempre van a ser individualistas, o tratan de ser individualistas, y cada quien, cada quien quiere desarrollar su trabajo independiente, o se asigna que uno hace una cosa y el otro la otra, pero la idea es tratar de enfocarlos para que lo hagan de manera colaborativa y que se logren mejores resultados". (docente, grupo focal).

Así, el docente, a través del rol mediador que ejerce dentro de las clases, motiva a los estudiantes para favorecer el trabajo en grupo y la interacción entre los mismos estudiantes. Esto posibilita la generación de cierto tipo de interacciones en las cuales los estudiantes superan sus dificultades y mejoran sus habilidades de comunicación. De hecho, una de las intervenciones de los docentes destaca la interacción entre los estudiantes como uno de los elementos del ambiente de aprendizaje que mejor se desarrollan en el ABP:

"La comunicación sí ha sido, digamos que ha venido mejorando, no, si mejorando en el sentido de que como son proyectos que ellos mismos proponen entonces la discusión no se da solamente en el entorno de la clase, sino también por fuera, porque ellos van con sus preguntas y entonces les surgirá mayor curiosidad, y buscan y como desde el inicio el trabajo ha sido actividades por grupos, ya discuten el trabajo”. (docente, grupo focal).

De acuerdo con Garret (2008), la implementación de estrategias de ABP permite que la interacción de los estudiantes se traslade a espacios informales, mejorando la relación entre los mismos y embebiéndolos en comunidades de aprendizaje mediante las cuales superan sus dificultades. El siguiente estudiante nos cuenta cómo la estrategia metodológica de $\mathrm{ABP}$ le ha enseñado a trabajar en equipo, adquirir responsabilidades y escuchar a sus compañeros:

"sí hemos aprendido, como a trabajar en grupo, como a tener una responsabilidad, ideas, o sea como a escuchar la opinión de todos y de eso sacar varias conclusiones, y trabajar en grupo, es prácticamente trabajar en grupo y convivir en el grupo". (estudiante, grupo focal).

Es posible afirmar entonces que la estrategia pedagógica implementada de ABP promueve ambientes de trabajo colaborativo, caracterizados porque los estudiantes se responsabilizan de su propio aprendizaje y asumen un rol indispensable para obtener las metas que sus grupos proponen. Es importante señalar que el ABP depende en gran medida de la generación de estas comunidades de aprendizaje, ya que son las que permiten que los estudiantes tomen un rol activo en la constitución del conocimiento.

\subsection{CLIMA DE RELACIONES}

El ABP genera un clima de relaciones en el cual los estudiantes pueden compartir ideas y reconocer el mérito de su trabajo. Esto es facilitado por el establecimiento de una relación horizontal entre estudiantes y docentes, la cual promueve un mejor clima de relaciones, permite un intercambio de ideas entre los diferentes actores en un espacio de confianza y compromete a estudiantes y docentes en la creación del conocimiento. 
"Yo siempre les he dicho: no miren al profesor como que yo me las sé todas y el día en el que yo no pueda responder una pregunta, "ay no, esa vieja no sabe nada", porque es que yo no me las sé todas. Todos venimos aquí a aprender. Yo sé algunas cosas, usted otras, usted me puede enseñar a mí, yo también le puedo enseñar a usted. Entonces debe ser un aprendizaje mutuo y quitarles esa idea de que el docente es el que sabe todo porque es que uno no se las sabe todas". (docente, grupo focal).

Este docente muestra cómo se pone en juego un ambiente de aprendizaje en el cual tanto profesores como estudiantes entran en un ciclo de actividades conjuntas y se apoyan mutuamente. De acuerdo con una de las docentes entrevistadas, la presencia de este tipo de clima de aula se encuentra relacionada con la apertura al conocimiento, el logro de los objetivos de clase y el compromiso frente a la misma:

"Yo he implementado otras estrategias, sino que en mi experiencia docente... y no, pienso que esta es la que permite que ellos sientan, sí participen, sí sean dinámicos, sí sean investigadores además porque potencializa muchas competencias que ellos deben tener, digamos, finalizando su ciclo escolar, que no la tienen de pronto en una clase tradicional, yo pienso también el hecho de que... les permite experimentar y de pronto fracasar es bueno porque en el colegio, digamos, el fracaso está mal visto. Digamos fracasar en una asignatura está como "en la casa me van a regañar", "me van a de pronto a pegar", pero digamos nunca se ha enfocado en que el fracasar también es aprender y entonces este tipo de metodologías permite eso". (docente, grupo focal).

El fragmento precedente evidencia cómo la implementación de la estrategia de ABP logra generar espacios en los cuales los estudiantes pueden discutir opiniones, enfrentar las propuestas de otros y probar nuevas ideas. Asimismo, la percepción de los estudiantes sobre el clima de relaciones muestra que estos se apropian y reconocen el espacio de aprendizaje como un lugar para intercambiar ideas y convertirse en agentes productores del conocimiento:

"Si todos nos tratamos bien en la clase, nos esforzamos por hacer, las cosas bonitas florecen, florecen, no es que es diferente uno hablar y exponer sus ideas a usted agarrarse y decir que no que es así, yo digo así es diferente darle la oportunidad a todos de que opinen”. (estudiante, grupo focal).

Los fragmentos precedentes ejemplifican cómo la implementación de la estrategia de ABP permitió el desarrollo de un clima de relaciones integrador-promotor, caracterizado por la presencia de un clima de confianza que permite el intercambio de ideas entre los estudiantes y profesores, y a su vez fomenta la búsqueda y exploración de diferentes objetivos y opciones de aprendizaje.

\subsection{SIGNIFICATIVIDAD}

Generar un interés genuino en los estudiantes por los contenidos de clase es una tarea que se ve facilitada en la medida en que dichos contenidos se vinculen con aspectos emocionales, afectivos, personales y contextuales de los estudiantes. No obstante, su implementa- 
ción se enfrenta a una dificultad inicial derivada del proceso de adaptación de los estudiantes al nuevo entorno de aprendizaje, ya que, según uno de los docentes, estos se encuentran acostumbrados a un carácter directivo en el aula de clase:

"Es complicado, para mí ha sido difícil porque hay grupos que realmente están negados y manifiestan directamente, profe por qué no volvemos a las clases anteriores que eran más divertidas, porque yo les acostumbraba a hacer guías y talleres y les marcaba qué era lo que tengo que hacer; entonces, de pronto es la educación que ellos han recibido, ellos han sido enseñados a hacer tareas puntuales ¿sí? a hacer tareas puntuales y ya una respuesta como el ABP es demasiado lenta para ellos. Ellos de una vez quisieran tener la identificación del problema y de una vez tener la solución, inclusive hay algunos que quieren entregar un producto sin interesarse si detrás de eso hay un conocimiento o hay desarrollo de alguna habilidad, simplemente lo quieren cambiar por una nota". (docente, grupo focal).

De esta manera, se ilustra cómo la metodología de ABP plantea espacios de aprendizaje en los cuales no existen respuestas o caminos únicos. Además se evidencian algunas de las resistencias que se encuentran en el aula de clase durante la implementación de la metodología. No obstante, dichas resistencias son transformadas en la medida en que los estudiantes tienen una motivación intrínseca hacia las actividades de clase y son capaces de desenvolverse en las mismas no por la nota, sino por los intereses mismos que el tema de trabajo les suscita.

"Pues yo pienso que como en todo ¿no? habrá estudiantes a los que les encanta y están súper motivados por el mismo tema que ellos escogieron, les encanta y van a hablar muy bien del proyecto y del área, y se sienten a gusto como habrá otros que ni les importa. La verdad es esa, sí, hay de todo, hay estudiantes que simplemente van porque el papá los obliga y hacen el proyecto ¡porque sí!”. (docente, grupo focal).

Esta intervención docente permite apreciar la interacción existente entre la temática de trabajo y la vinculación emocional y afectiva de los estudiantes con el aprendizaje. Asimismo, uno de los estudiantes resalta que la utilidad percibida de los conocimientos de la clase de tecnología aumenta cuando dichos conocimientos pueden ser aplicados para la resolución de problemas reales en su entorno:

"Y por eso mismo, porque pues al final empezamos, o sea esos conocimientos no quedan simplemente ahí, sino los podemos aplicar para ayudar a la comunidad ¿no? a solucionar los problemas". (estudiante, grupo focal).

De este modo, se dilucida la manera en que la implementación de la metodología les brinda a los estudiantes contenidos que les son cercanos emocionalmente y les hacen explícita la utilidad de los conocimientos que son adquiridos en el aula de clase. Por otro lado, escasean ejemplos que muestren que los estudiantes vinculan los nuevos aprendizajes con experiencias o conocimientos previos. Esto puede estar motivado por la falta de una colaboración interdisciplinaria en la elaboración de los proyectos, la cual implanta la idea en los estudiantes de una ruptura entre las diferentes materias. Al respecto, el siguiente 
profesor nos muestra como las condiciones estructurales de la configuración del colegio y el aula de clase necesitan ser cambiadas, de tal manera que se supere el fraccionamiento percibido en los estudiantes con respecto a los conocimientos adquiridos en el proyecto:

\begin{abstract}
"Yo pienso que también influye mucho la parte administrativa, porque ese trabajar por proyectos interdisciplinarios exige que se rompa el esquema de horario, que se rompa el esquema de que tengo que tener a los estudiantes allá metidos en el salón y cuidadito se salen o hacen desorden, lo que implica un poquito de desorden académico y eso nuestros directivos no lo ven tan bien, entonces por un lado falta eso. Por otro lado, pues también que algunos profesores trabajan bajo el esquema de que tengo que dictar estos temas y son estos temas y si no, no cumplo con la programación del año, no se dan cuenta de que con un proyecto como que le encuentran más significado los muchachos a esos contenidos que uno plantea para las sesiones". (docente, grupo focal).
\end{abstract}

En conclusión, se percibe que la implementación de la metodología modifica la visión del conocimiento que tienen profesores y estudiantes, mostrándoles que tal conocimiento les permite responder a diferentes problemáticas. Además, es importante destacar que el ABP logra que los estudiantes se vinculen afectivamente con los contenidos de la materia, pues introduce en el aula objetivos enfocados en el dominio real de los contenidos. De este modo, se promueve un entorno de aprendizaje centrado en los estudiantes que cambia el sistema de castigos y recompensas por uno en el cual se promueve una motivación intrínseca a partir de actividades que por sí mismas les puedan interesar al estudiante.

\title{
6.5. COMPETENCIAS TECNOLÓGICAS
}

La literatura alrededor del ABP ha mostrado que esta metodología reúne las características necesarias para desarrollar en los estudiantes habilidades de pensamiento de orden superior (indagación, creatividad y pensamiento crítico). Así se ve demostrado en las intervenciones de los profesores y estudiantes, las cuales ilustran los beneficios de la estrategia de ABP sobre el desarrollo de nuevas habilidades y competencias en los alumnos. El primer efecto se encuentra en los contenidos temáticos de la clase misma, los cuales, según varios de los docentes, venían orientados hacia conocimientos básicos en ofimática y fueron extendidos a la tecnología:

"Sí ha sido un poco complejo para nosotros porque nosotros no veníamos trabajando tecnología sino informática como tal, entonces le metieron los conceptos nuevos que los chicos no los manejan. Es decir, allá siempre hemos trabajado informática, no tecnología, y es muy diferente, son muy diferentes las dos". (docente, grupo focal).

Este ejemplo destaca que el primer cambio que ocurre con la implementación del programa es la concepción misma de la naturaleza de la tecnología, la cual en los estudiantes y profesores venía ligada a la ofimática o al uso de herramientas tecnológicas. Tales cambios permitieron que tanto docentes como estudiantes replantearan los objetivos de la clase, enfocándose en crear usuarios cultos y críticos de la tecnología que puedan hacer uso de esta para la solución de los problemas que encuentran en su contexto. Así se evidencia en las siguientes intervenciones: 
"el uso adecuado es enseñarle al estudiante hacer uso adecuado de todas las tecnologías de su contexto. Hacerle un uso racional a toda esa tecnología, un uso crítico y que esté en capacidad de transformar las cosas que él considera que están afectando no solamente su contexto, sino todo un sistema, porque hay comportamientos de cultura que en el uso de las tecnologías afectan no solo a su familia, su institución sino a toda una ciudad. Entonces es importante desarrollar en el estudiante, a través de las competencias tecnológicas... de hacer uso adecuado para hacer uso a su alrededor". (docente, grupo focal).

"Cuando piensa en tecnología, se le vienen así como, como decirlo, como cosas de información, no sé qué, pero pues es importante tener en cuenta que no solamente es eso, sino que es como aprender a usar, y pues es importante, porque en estas épocas, pues eso es lo que más se usa ino!, entonces pues ahí es importante aprenderlo, usarlo, y sobretodo ahorita como estamos con lo del proyecto, pues ayudar a la comunidad, ayudar a los demás para mejorar todo”. (estudiante, grupo focal).

Se puede apreciar que la implementación de la estrategia enfoca la clase de tecnología en lograr que los estudiantes sean capaces de hacer uso de las herramientas tecnológicas para solucionar los problemas de su contexto. La metodología ABP facilita esto, ya que mediante esta los estudiantes aprenden a desarrollar sus capacidades para identificar problemas, analizar diferentes soluciones y usar la tecnología para resolverlos:

"yo creo que lo que estamos haciendo nos sirve bastante, porque nosotros estamos aprendiendo a resolver problemas, a darle más de una respuesta a ese problema y tener varias alternativas, digamos cuando nosotros ya estemos mayores, digamos cuando tengamos un problema que tengamos que estudiar, digamos va a ser más fácil darle una respuesta”. (estudiante, grupo focal).

Se evidencia además que los estudiantes desarrollaron las competencias tecnológicas de apropiación, uso de la tecnología y solución de problemas, las cuales fueron adaptadas de los lineamientos de la Guía 30 del Ministerio de Educación Nacional teniendo en cuenta las categorías de Bloom (1956) (Ver Tabla 3). 
Tabla 3. Reportes de estudiantes que evidencian cumplimiento de desempeños en las competencias de Apropiación y Uso de la Tecnología y Solución de Problemas

\begin{tabular}{|c|c|c|}
\hline Competencia & Desempeño & Ejemplo \\
\hline \multirow{8}{*}{$\begin{array}{l}\text { Apropiación } \\
\text { y Uso de la } \\
\text { Tecnología }\end{array}$} & $\begin{array}{|lr|}\text { Reconozco la } & \text { importancia } \\
\text { del mantenimiento de } & \text { artefactos r tecnológicos } \\
\text { utilizados en la vida cotidiana }\end{array}$ & $\begin{array}{l}\text { “...pues porque a la hora de hacer las diferentes actividades tenía } \\
\text { uno que, para no dañar los artefactos, pues hacerles mantenimiento } \\
\text { y saber qué tipo de mantenimiento se le hacía, por decir a la cámara } \\
\text { cuando grabamos, el computador cuando lo utilizamos" }\end{array}$ \\
\hline & $\begin{array}{l}\text { Consulto y documento } \\
\text { información sobre algunos } \\
\text { procesos de } \\
\text { manufactura de producción y }\end{array}$ & $\begin{array}{l}\text { “...pues yo creo que, pues es que cuando investigamos sobre el } \\
\text { proceso, en el proyecto de nosotros, pues supimos pues, qué era, } \\
\text { ya como habíamos visto harto sobre la tabla y todo eso, sabíamos } \\
\text { ya prácticamente las consecuencias, causas y todo" }\end{array}$ \\
\hline & $\begin{array}{l}\text { Utilizo adecuadamente her- } \\
\text { ramientas de las TIC para la } \\
\text { búsqueda, procesamiento y } \\
\text { socialización de la infor- } \\
\text { mación }\end{array}$ & $\begin{array}{l}\text { "Pues es que yo, pues el ejemplo de Kevin por ejemplo de las } \\
\text { exposiciones, a mí me gusta hacer como muchos esquemitas y no } \\
\text { solo texto, entonces saber cómo se utiliza cómo se hace. Además, } \\
\text { a mí me gusta esa parte de mirar programas, saber cómo parte } \\
\text { más, como tecnológica no sé, como de sistemas, sí un poquito de } \\
\text { sistemas entonces pues como que se me hacía más fácil } \\
\text { organizarlo y también con los programas, como de hacer } \\
\text { esquemitas y eso que ustedes nos daban entonces se nos hacía } \\
\text { más fácil" }\end{array}$ \\
\hline & $\begin{array}{l}\text { Utilizo elementos de protec- } \\
\text { ción en ambientes de trabajo } \\
\text { y de producción teniendo en } \\
\text { cuenta las normas de seguri- } \\
\text { dad industrial }\end{array}$ & $\begin{array}{l}\text { “...pues sí porque, porque cuando yo por ejemplo necesito, } \\
\text { necesito hacer por ejemplo, no sé, arreglar un cable o manipular } \\
\text { cosas eléctricas pues necesito guantes aislante, overoles o cosas } \\
\text { así, por seguridad”. }\end{array}$ \\
\hline & $\begin{array}{l}\text { Interpreto y utilizo manuales, } \\
\text { instrucciones, diagramas y } \\
\text { esquemas, para el montaje de } \\
\text { algunos artefactos, dispositi- } \\
\text { vos y sistemas tecnológicos }\end{array}$ & $\begin{array}{l}\text { "...él nos muestra un esquema y entonces ya uno como que } \\
\text { intelectualmente ya le graba y uno sabe qué es lo que tiene que } \\
\text { hacer, los pasos a seguir para eso por ejemplo en Corel llega y nos } \\
\text { explica las herramientas y nosotros ya sabemos para qué sirve } \\
\text { cada herramienta para trabajarlo" }\end{array}$ \\
\hline & $\begin{array}{l}\text { Utilizo herramientas y equi- } \\
\text { pos en la construcción de } \\
\text { modelos, maquetas o prototi- } \\
\text { pos, teniendo en cuenta nor- } \\
\text { mas de seguridad }\end{array}$ & $\begin{array}{l}\text { "pues en las pruebas, pues realmente esas pruebas nos ayudaron } \\
\text { pues a tener más conocimientos y pues el uso de herramientas e } \\
\text { instrumentos para la creación de prototipos, maquetas y modelos } \\
\text { siguiendo las normas de seguridad es como, yo quiero crear, crear } \\
\text { una maqueta y de acuerdo a eso yo miro qué herramientas debo } \\
\text { utilizar, cómo me debo proteger para no causarme daños con esos } \\
\text { instrumentos y pues cómo debo crearla, pues las medidas y cómo } \\
\text { debe ser de grande" }\end{array}$ \\
\hline & $\begin{array}{l}\text { Utilizo el trabajo en equipo } \\
\text { como estrategia para realizar } \\
\text { proyectos tecnológicos }\end{array}$ & $\begin{array}{l}\text { "Cumple con las funciones que me asignan dentro de un equipo, } \\
\text { porque siento que hacemos los grupos y escogemos siempre, tengo } \\
\text { esa, ese compromiso con ellos y consigo misma de que si me } \\
\text { propongo algo lo tengo que cumplir, sea como sea, pero tengo que } \\
\text { cumplir siempre y pues me esfuerzo para organizarse" }\end{array}$ \\
\hline & $\begin{array}{l}\text { Identifico y explico la rel- } \\
\text { ación de conocimientos y } \\
\text { conceptos de otras discipli- } \\
\text { nas involucradas en el fun- } \\
\text { cionamiento de algunos arte- } \\
\text { factos }\end{array}$ & $\begin{array}{l}\text { No hay ejemplos relevantes. Esta categoría no se logra desarrollar } \\
\text { debido a la falta de trabajo interdisciplinario que se dio en esta } \\
\text { intervención }\end{array}$ \\
\hline
\end{tabular}




\begin{tabular}{|c|c|c|}
\hline \multirow{6}{*}{$\begin{array}{l}\text { Solución de } \\
\text { Problemas }\end{array}$} & $\begin{array}{l}\text { Identifico las condiciones, } \\
\text { especificaciones y restric- } \\
\text { ciones de diseño utilizadas } \\
\text { para la solución de un proble- } \\
\text { ma tecnológico }\end{array}$ & $\begin{array}{l}\text { "La mayoría del tiempo uno ve como 'esta cosa se puede hacer } \\
\text { porque tal cosa es viable' o "esto no se puede hacer porque hay } \\
\text { algo que lo hace complicado"". }\end{array}$ \\
\hline & $\begin{array}{l}\text { Propongo, analizo y comparo } \\
\text { diferentes soluciones a un } \\
\text { mismo problema, explicando } \\
\text { su origen, ventajas y dificul- } \\
\text { tades }\end{array}$ & $\begin{array}{l}\text { “...digamos mi proyecto es sobre el riego de plantas, entonces } \\
\text { nosotros decíamos ¡no, no, no! vamos a usar aspersores y después } \\
\text { aparecía que hay unas plantas que no pueden recibir cierta } \\
\text { cantidad de agua. Entonces se va estructurando una solución } \\
\text { como más viable y más certera para un problema, eso me pareció } \\
\text { que es muy valioso y es algo que de verdad durante este semestre } \\
\text { desarrollé bastante, y me parece que es una excelente habilidad a } \\
\text { destacar porque le permite a uno como digamos, generar una } \\
\text { perspectiva de un problema, y a partir de las diferentes situaciones } \\
\text { o los diferentes como tapujos que se le presentan en un problema } \\
\text { a uno, uno puede dar una solución y puede ser muy acertada y } \\
\text { muy certera. Eso fue lo que me gustó” }\end{array}$ \\
\hline & $\begin{array}{l}\text { Interpreto y represento ideas } \\
\text { sobre diseños, innovaciones } \\
\text { o protocolos de experimentos } \\
\text { mediante el uso de registros, } \\
\text { textos, diagramas, figuras, } \\
\text { planos constructivos, maque- } \\
\text { tas, modelos y prototipos, } \\
\text { empleando para ello (cuando } \\
\text { sea posible) TIC }\end{array}$ & $\begin{array}{l}\text { “...hicimos varias como construcciones en los programas de } \\
\text { Sketchup y eso, digamos hicimos un plano de la cafetería, } \\
\text { hicimos las mediciones en físico y después las pasamos a } \\
\text { computador. Y, pues eso nos ayudó como, manejarlo desde varios } \\
\text { ángulos y, pues, se nos hizo como fácil en mi grupo" }\end{array}$ \\
\hline & $\begin{array}{l}\text { Diseño, construyo y pruebo } \\
\text { prototipos de artefactos, } \\
\text { sistemas y procesos (como } \\
\text { respuesta a necesidades o } \\
\text { problemas), teniendo en } \\
\text { cuenta las restricciones y es- } \\
\text { pecificaciones planteadas }\end{array}$ & $\begin{array}{l}\text { "Pues mi idea fue de presentación, video de diseño, ya que a mí } \\
\text { me gusta harto eso de la programación, de eso de programas de } \\
\text { sketchear para hacer, en todo eso, ya que yo en el grupo fui el que } \\
\text { cogí todo el diseño de computadores, todo cualquier cosa esa de } \\
\text { tecnología [¿?] [E1: Tú fuiste el que hizo eso] Ujum, por eso fue } \\
\text { que esa fue mi habilidad de diseño y eso, de lo de maquetas" }\end{array}$ \\
\hline & $\begin{array}{l}\text { Evalúo y selecciono con ar- } \\
\text { gumentos mis propuestas y } \\
\text { decisiones en torno a un } \\
\text { diseño }\end{array}$ & $\begin{array}{l}\text { “...o sea, nosotros damos respuestas y en torno a ellas hacemos } \\
\text { el proyecto de, por ejemplo, de crear pasto, entonces a nosotros } \\
\text { nos dijeron que diferentes semillas o algo así, de cual era más } \\
\text { barato o algo así, acá íbamos y las escogimos" }\end{array}$ \\
\hline & $\begin{array}{l}\text { Propongo mejoras a solu- } \\
\text { ciones tecnológicas argu- } \\
\text { mentando los criterios y la } \\
\text { ponderación de las restric- } \\
\text { ciones utilizadas }\end{array}$ & $\begin{array}{l}\text { "...O sea buscar un problema que las demás personas ya hayan } \\
\text { tratado de solucionar o que, por el contrario, no sé, no, no requería } \\
\text { la mayor importancia, y nosotros lo trabajamos. Y tratamos de } \\
\text { solucionarlo con las TIC" }\end{array}$ \\
\hline
\end{tabular}

Estas intervenciones nos permiten ver el impacto que tuvo la implementación del programa en el desarrollo de las diferentes competencias tecnológicas de los estudiantes que se promueven en la Guía 30 del MEN (MEN, 2008), alcanzándose así los objetivos de este programa de intervención.

En resumen, los resultados desplegados muestran que los ambientes de aprendizaje producto de las estrategias de ABP no solo tienen efectos en el aprendizaje de contenidos, 
sino que también desarrollan otros elementos que son primordiales para lograr los objetivos de la alfabetización tecnológica: significatividad, rol docente, interacción entre estudiantes, clima de relaciones y competencias tecnológicas.

\section{DISCUSIÓN Y CONCLUSIONES}

La literatura existente alrededor de las metodologías de aprendizaje centradas en el estudiante señala la necesidad de un cambio radical con respecto a la forma de enseñanza y aprendizaje que se da tradicionalmente en las aulas de clase. Los resultados de esta investigación ejemplifican algunos de los campos en los cuales dichos cambios ocurren, incluyendo aspectos como el rol docente, la interacción entre estudiantes, el clima del aula, la significatividad y las competencias tecnológicas.

El ABP como representante de las metodologías de aprendizaje centradas en el estudiante destaca la importancia de cambiar el rol de estudiantes y profesores en la construcción del conocimiento, permitiendo así que los estudiantes tengan un aprendizaje auto-regulado (Smith et al., 2014). No obstante, en esta investigación se resalta como un factor clave en la consecución de tales objetivos el rol docente. En particular porque es este quien a través de procesos de acompañamiento y supervisión asegura que los estudiantes superen las dificultades que se les presentan y se comprometan en las actividades de aprendizaje que ellos mismos han definido. De esta manera, exploramos diversos caminos que nutren los postulados de Thomas (2000) sobre la función del rol docente en la implementación del ABP.

Los resultados además soportan los postulados de Garrett (2008), pues evidencian que la implementación de ambientes de aprendizaje centrados en el estudiante promueven la interacción entre alumnos y modifican la relación alumno profesor, permitiendo el desarrollo de nuevas habilidades de pensamiento superior y la conformación de comunidades de aprendizaje.

Con respecto a los retos que enfrentan los estudiantes en la implementación del ABP señalados por Hung (2011), vemos que pueden ser superados dándoles la libertad a los estudiantes de elegir un tema de investigación que sea aplicable en la resolución de un problema de su contexto inmediato. Según Heydrich et al. (2012), dicha acción genera una motivación intrínseca en los estudiantes y permite la inclusión de elementos que hacen parte de la vida cotidiana de los alumnos.

Por último, los resultados muestran que la implementación de la metodología de ABP en la enseñanza de la tecnología tiene efectos en el desarrollo de competencias y habilidades tecnológicas. Esto se evidencia en el cambio de estudiantes y profesores sobre la naturaleza de la tecnología, la cual solía ligarse a ideas relacionadas con la ofimática (Jones et al., 2013). Dicho cambio fue crucial en esta intervención para la implementación de objetivos educativos acordes a los objetivos de la alfabetización tecnológica.

De esta manera, quedan demostradas las ventajas de permitir nuevos espacios educativos, los cuales aportan en la creación de sociedades innovadoras favoreciendo el desenvolvimiento del individuo en actividades de diseño, exploración, identificación de problemas, construcción, modelamiento, reparación y evaluación en contextos de aprendizaje significativos. Así, estos escenarios se constituyen en espacios importantes para el desarrollo de las capacidades y habilidades que se encuentran implicadas en la alfabetización tecnológica. 
En conclusión, esta investigación evidencia que la implementación de la metodología de ABP permite que se desarrollen competencias y habilidades necesarias en los estudiantes para que identifiquen problemas, propongan soluciones y tomen decisiones a través del uso de la tecnología. Todo lo anterior es motivado por una serie de cambios en el ambiente de aprendizaje que no solo afectan los contenidos de clase, sino que además comprenden cambios en el rol docente, la interacción entre estudiantes, el clima del aula y la significatividad.

Se consolida así un desafío para las instituciones educativas, en tanto se demanda la necesidad de instituciones más flexibles que permitan el desarrollo de trabajos interdisciplinarios y que incluyan en su seno a la sociedad y las problemáticas que los rodean. Todo ello implica un reto institucional y cultural que renueve y repiense los lineamientos con los cuales la sociedad ve la educación, permitiendo equilibrar los contenidos curriculares con las realidades contextuales por las cuales atraviesan tanto los profesores como los estudiantes. Así, el alcance de los objetivos de la alfabetización tecnológica dependerá de la apertura cultural y la innovación educativa.

\section{REFERENCIAS BIBLIOGRÁFICAS}

Acar, G. (2013). The effect of Project-Based learning on student's motivation. International Journal Of Academic Research, 5(2), 82-86. doi:10.7813/2075-4124.2013/5-2/B.11

Ames, C. (1992). Classrooms: goals, structures, and student motivation. Journal of Educational Psychology, 84, 261-271.

Australian Education Council. (1994). A statement on technology for Australian schools, A joint project of the States, Territories and the Commonwealth of Australia initiated by the Australian Education Council. Carlton, Victoria, Australia: Curriculum Corporation.

Bloom, B. S. (1956). Taxonomy of educational objectives. Vol. 1: Cognitive domain. New York: McKay.

Castañeda, M., \& Corredor, J. (2014). Las nuevas tecnologías y el sujeto docente: Incidencia de las relaciones con las nuevas tecnologías en docentes colombianos y en sus prácticas. En Ochoa, L. (Ed.), Investigación e innovación educativas: docentes (pp. 141-173). Bogotá: Centro Editorial de Facultad de Ciencias Humanas de la Universidad Nacional de Colombia.

Devlin, T., Feldhaus, C., \& Bentrem, K. (2013). The evolving classroom: A study of traditional and technology-based instruction in a STEM classroom. Journal of Technology Education, 25(1), 34-54.

de Vries, M. J. (1999). Developing technology education by learning from other countries and from industry. In T. Kananoja, J. Kantola, \& M. Issakainen (Dds.), Development of technology education-Conference -98 (pp. 137-149). University of Jyväskylä. Department of Teacher Education. The Principles and Practice of Teaching 33.

Edwards, C. (2004). Clasroom management and discipline. New York: Jhon Wiley \& Sons.

English, M. C., \& Kitsantas, A. (2013). Supporting Student Self-Regulated Learning in Problem-and Project-Based Learning. Interdisciplinary Journal Of Problem-Based Learning, 7(2), 128-150.

Esteban, M. (2002). El diseño de entornos de aprendizaje constructivista. RED. Revista de Educación a Distancia, 6. Recuperado de http://www.redalyc.org/articulo.oa?id=54700601

Freiberg, H.J. (1999). Beyond behaviorism: Changing the classroom management paradigm. Boston: Allyn and Bacon.

Fox-Turnbull, W. (2012). Learning in Technology. In P. Williams (Ed.), Technology education for teacher (pp. 55-92). Rotterdam: SensePublishers. 
Garrett, T. (2008). Student-centered and teacher-centered classroom management: A case study of three elementary teachers. The Journal of Classroom Interaction, 43(1), 34-47.

Grant, M. M., \& Hill, J.R. (2006). Weighing the rewards with the risks? Implementing student-centered pedagogy within high-stakes testing. In R. Lambert \& C. McCarthy (Eds.), Understanding teacher stress in the age of accountability (pp. 19-42). Greenwich, CT: Information Age.

Hannafin, M. J., \& Land, S. M. (1997). The foundations and assumptions of technology-enhanced student-centered learning environments. Instructional science, 25(3), 167-202.

Heydrich, M., Rojas, M., \& Hernández, A. (2012). Aprendizaje basado en proyectos: una experiencia de innovación docente. Revista Universidad EAFIT, 46(158), 11-21.

Hung, W. (2011). Theory to reality: A few issues in implementing problem-based learning. Educational Technology Research and Development, 59(4), 529-552. doi:10.1007/s11423-0119198-1

ITEA/ITEEA. (2000). Standards for technological literacy: Content for the study of technology. Reston, VA: Author.

ITEA/ITEEA. (2002). Standards for technological literacy: Content for the study of technology. Reston, VA: Author.

ITEA/ITEEA. (2007). Standards for technological literacy: Content for the study of technology. Reston, VA: Author.

Jonassen, D. (2000). El diseño de entornos constructivista de aprendizaje. En Ch. Reigeluth (Ed.), Diseño de la instrucción: teoría y modelos (pp. 225-250). Madrid: Santillana.

Jones, A., Buntting, C., \& de Vries, M. J. (2013). The developing field of technology education: A review to look forward. International Journal of Technology and Design Education, 23(2), 191212.

Krajcik, J. S., \& Blumenfeld, P. (2006). Project-based learning. In R. K. Sawyer (Ed.), The Cambridge handbook of the learning sciences (pp. 317-334). New York: Cambridge.

Krajcik, J., Blumenfeld, P. C., Marx, R. W., Bass, K. M., Fredricks, J., \& Soloway, E. (1998). Inquiry in project-based science classrooms: Initial attempts by middle school students. Journal of the Learning Sciences, 7(3-4), 313-350.

Krajcik, J., Blumenfeld, P. C., Marx, R. W., \& Soloway, E. (1994). A collaborative model for helping middle grade science teachers learn project-based instruction. The elementary school journal, 94(5), 483-497. doi:10.1086/461779

Lee, D., Huh, Y., \& Reigeluth, C. M. (2015). Collaboration, intragroup conflict, and social skills in project-based learning. Instructional Science, 43(5), 561-590.

Lee, J. S., Blackwell, S., Drake, J., \& Moran, K. A. (2013). Taking a Leap of Faith: Redefining Teaching and Learning in Higher Education Through Project-Based Learning. Interdisciplinary Journal of Problem-based Learning, 8(2), 19-34. doi:10.7771/1541-5015.1426

Madrigal, C. M., Mujica, A. D., Tamarín, C. C., Olave, C. N., \& Carrasco, I. B. (2011). Clima social escolar en el aula y vínculo profesor-alumno: Alcances, herramientas de evaluación, y programas de intervención. Revista Electrónica de Psicología Iztacala, 14(3), 70-84.

Marzano, R.J. (2001). Designing a new taxonomy of educational objectives. Thousand Oaks, CA: Corwin.

Ministerio de Educación Nacional. (2008). Ser competente en tecnología: juna necesidad para el desarrollo! Orientaciones generales para la educación en tecnología (MEN). Bogotá: Autor. Recuperado de http://www.mineducacion.gov.co/1759/articles-160915_archivo_pdf.pdf

Miles, M. B., Huberman, A. M., \& Saldaña, J. (2013). Qualitative data analysis: A methods sourcebook. Thousand Oaks, CA: SAGE Publications, Incorporated.

Muñoz, F. O., Arvayo, K. L., Villegas, C. A., González, F. H., \& Sosa, O. A. (2014). El método colaborativo como una alternativa en el trabajo experimental de Química Orgánica. Educación química, 25(4), 464-469.

Namey, E., Guest, G., Thairu, L., \& Johnson, L. (2008). Data reduction techniques for large qualitative 
data sets. In G. Guest \& K. M. MacQueen (Eds.), Handbook for team-based qualitative research (pp. 137-162). Lanham, MD: Rowman \& Littlefield.

Neo, M., \& Neo, T.-K. (2009). Engaging students in multimedia-mediated constructivist learningStudents' perceptions. Educational Technology \& Society, 12(2), 254-266.

Oxfam, I. (2009). Pistas para cambiar la escuela. España: Intermón-Oxfam.

Panasan, M., \& Nuangchalerm, P. (2010). Learning Outcomes of Project-Based and Inquiry-Based Learning Activities. Online Submission, 6(2), 252-255.

Patton, M.Q. (2001). Qualitative Evaluation and Research Methods (2nd ed.). Thousand oaks, CA: Sage Publications.

Qualifications and Curriculum Authority. (2000). Design and technology in the National Curriculum. London: HMSO.

Rasinen, A. (2003). An analysis of the technology education curriculum of six countries. Journal of Technology Education, 15(1), 31-47.

Reverte, J., Gallego, A. J., Molina, R., \& Satorre, R. (2007). El aprendizaje basado en proyectos como modelo docente. Experiencia interdisciplinar y herramientas groupware. Actas de las XIII Jornadas de Enseñanza Universitaria de la Informática, 2007.

Rugeles, P., Mora, B., Metaute, P., \& Díaz, J. (2014). El trabajo colaborativo en la educación superior mediadas por las tecnologías de la información. Global Conference on Business and Finance Proceedings, 9(1), 1551-1559.

Saldana, J. (2009). The coding manual for qualitative researchers. Thousand Oaks, CA: Sage.

Smith, K., de Brabander, C. J., \& Martens, R. L. (2014). Student-centred and teacher-centred learning environment in pre-vocational secondary education: Psychological needs, and motivation. Scandinavian Journal of Educational Research, 58(6), 695-712.

Tamim, S. R., \& Grant, M. M. (2013). Definitions and Uses: Case Study of Teachers Implementing Project-based Learning. Interdisciplinary Journal of Problem-Based Learning, 7(2), 72-101. doi:10.7771/1541-5015.1323

Thomas, J.W. (2000). A review of research on project-based learning. San Rafael, CA: Autodesk Foundation.

Vratulis, V., Clarke, T., Hoban, G., \& Erickson, G. (2011). Additive and disruptive pedagogies: The use of slowmation as an example of digital technology implementation. Teaching and Teacher Education, 27(8), 1179-1188. doi:10.1016/j.tate.2011.06.004

Wu, H. K., \& Huang, Y. L. (2007). Ninth-grade student engagement in teacher-centered and studentcentered technology-enhanced learning environments. Science Education, 91(5), 727-749.

Wurdinger, S., \& Qureshi, M. (2015). Enhancing College Students' Life Skills through Project Based Learning. Innovative Higher Education, 40(3), 279-286. 